\title{
Vaccine manufacturing: challenges and solutions
}

\author{
Jeffrey B Ulmer ${ }^{1}$, Ulrich Valley ${ }^{2} \&$ Rino Rappuoli $^{3}$
}

The recent influenza vaccine shortages have provided a timely reminder of the tenuous nature of the world's vaccine supply and the potential for manufacturing issues to severely disrupt vital access to important vaccines. The application of new technologies to the discovery, assessment, development and production of vaccines has the potential to prevent such occurrences and enable the introduction of new vaccines. Gene-based vaccines, virus-like particles, plant-derived vaccines and novel adjuvants and delivery systems represent promising approaches to creating safer, more potent vaccines.

을 As a consequence, more people will have faster access to more effective vaccines against a broader spectrum of infectious diseases. However, the increased cost of producing new vaccines and regulatory uncertainty remain challenges for vaccine manufacturers.

The prevention of diseases by vaccination is without question one of the most significant medical achievements of mankind. Vaccines currently prevent more than 3 million deaths per year, and the positive economic impact is in excess of a billion dollars per year. During the 20th century, the average human life span has increased by approximately 30 years, a significant portion of which has been attributed directly to vaccination ${ }^{1}$. One infectious disease, smallpox, has been eradicated by vaccination, and another, polio, is expected to be eradicated. However, the stringent regulatory hurdles associated with immunizing healthy people and the perception of modest financial returns on investment have presented a high barrier for entry of pharmaceutical companies into the vaccine business. As a consequence, the number of vaccine manufacturers has decreased in recent years, leading to less competition and little incentive to invest in new technologies ${ }^{2}$.

The landscape seems to be changing, however. Several new vaccines have recently been licensed for human use, including some that are expected to achieve blockbuster status ( $>\$ 1$ billion in annual sales), such as Prevnar (Wyeth Pharmaceuticals) for pneumococcal disease and Gardasil (Merck) for human papillomavirus (HPV) infection. These scientific and economic successes, and the potential for more, have led to the expectation that the vaccine business will grow at a greater rate than the pharmaceutical drug business over the next decade ${ }^{3-5}$. If so, the concomitant development and application of innovative technologies

\footnotetext{
${ }^{1}$ Novartis Vaccines and Diagnostics, 4560 Horton St., Mailstop 4.3, Emeryville, California 94608, USA. ${ }^{2}$ Novartis Vaccines and Diagnostics, GmbH \& Co. KG, Emil von Behring Strasse 76, D-35041 Marburg, Germany. ${ }^{3}$ Novartis Vaccines and Diagnostics S.r.I., Via Fiorentina No. 1, I-53100, Siena, Italy. Correspondence should be addressed to J.B.U. (jeffrey_ulmer@chiron.com).
}

Published online 8 November 2006; doi:10.1038/nbt1261 should increase the chances of success for other important new vaccines (such as those for HIV, malaria and hepatitis C).

Vaccination was first implemented on a wide scale more than 200 years ago with the introduction of the smallpox vaccine. Today, many infectious diseases caused by various viruses and bacteria can be prevented by vaccination (Table 1 ). However, infectious diseases still cause substantial morbidity and mortality, so many new or improved vaccines are needed. To this end, certain suboptimal vaccines have been replaced with better versions, and new vaccines are being introduced at a higher frequency than ever before. Half of all new vaccines have been developed in the past 25 years, at a rate of approximately one per year over that time frame, compared to an average of one every 5 years before that. These new and improved vaccines have resulted in changes in the way vaccines are manufactured; in some instances, manufacturing issues themselves have provided the driver for these changes.

This review will include an overview of the main types of vaccines available today and how they are manufactured, examples of how certain vaccines have undergone changes in the way they are manufactured, and how new technologies may revolutionize the way vaccines will be made in the future.

\section{Vaccine types}

The major types of vaccines licensed for use in humans include live, attenuated organisms and killed or inactivated organisms. Fewer vaccines are based on partially purified components of an organism, and there are a handful of recombinantly produced subunit vaccines (see Table 1).

Live, attenuated organisms. The smallpox vaccine-the first vaccine developed for widespread use - consisted of a live bovine poxvirus that is closely related to the smallpox virus but unable to induce disease in humans. This concept of using live organisms to elicit protective immunity was subsequently adopted for vaccines against several bacterial and viral pathogens, such as Vibrio cholerae, Mycobacterium tuberculosis, Salmonella typhi, yellow fever virus, measles virus, mumps virus, poliovirus, rubella virus, varicella-zoster virus, adenovirus and rotavirus.

The success of this approach depends on decoupling virulence from induction of protective immunity. Once this has been achieved, live, attenuated organisms are very effective as vaccines because they contain the three main elements needed for potent immune induction: (i) target antigen(s) for memory responses, (ii) pathogen-associated molecular patterns for stimulation of innate immunity (that is, adjuvant effects) and (iii) efficient delivery resulting from the natural invasiveness of the organism (Fig. 1). In effect, the immune system treats live, attenuated vaccines as it would an infectious pathogen. Methods used to derive safe versions of pathogenic organisms generally involve the induction and selection of mutants to reduce or eliminate virulence. For example, the 
Table 1 Main types of vaccines

\begin{tabular}{|c|c|c|}
\hline Vaccine type & Selected disease targets & Vaccine preparation \\
\hline \multirow[t]{7}{*}{ Live attenuated } & Smallpox & Crude preparation of cowpox infected calf skin \\
\hline & Tuberculosis & Mycobacterium bovis BCG grown in media \\
\hline & Yellow fever & Purified, attenuated virus grown in eggs \\
\hline & Polio & Purified, attenuated virus grown in tissue culture cells \\
\hline & Chickenpox & Purified, attenuated virus grown in tissue culture cells \\
\hline & Rotavirus & Purified, attenuated virus grown in tissue culture cells \\
\hline & Influenza & Purified, attenuated virus grown in eggs \\
\hline \multirow[t]{6}{*}{ Killed } & Typhoid fever & Inactivated Salmonella typhi grown in media \\
\hline & Plague & Inactivated Yersinia pestis grown in media \\
\hline & Whooping cough & Inactivated whole-cell Bordetella pertussis grown in media \\
\hline & Influenza & Inactivated virus grown in eggs \\
\hline & Polio & Inactivated virus grown in tissue culture cells (see Table 2 ) \\
\hline & Hepatitis A & Inactivated virus grown in tissue culture cells \\
\hline \multirow[t]{7}{*}{ Purified subunit } & Diphtheria & Inactivated toxin from Corynebacterium diphtheriae grown in media \\
\hline & Tetanus & Inactivated toxin from Clostridium tetani grown in media \\
\hline & Pneumococcus & Polysaccharides from 23 Streptococcus pneumoniae strains grown in media \\
\hline & Meningococcus & Polysaccharides from four Neisseria meningitidis strains grown in media \\
\hline & Haemophilus influenzae $B$ & Polysaccharides from $H$. influenzae chemically conjugated to carrier protein (see Table 2) \\
\hline & Pertussis & Acellular extract of $B$. pertussis grown in media (see Table 2) \\
\hline & Anthrax & Culture supernatant of Bacillus anthracis grown in media \\
\hline \multirow[t]{2}{*}{ Recombinant subunit } & Hepatitis B & Purified, recombinant HBsAg VLP produced in tissue culture cells (see Table 2) \\
\hline & Borrelia burgdorferi & Purified, recombinant OspA protein produced in tissue culture cells (no longer available) \\
\hline
\end{tabular}

S. typh $i$ vaccine strain Ty21a was generated from wild type using mutagenic agents, resulting in several mutated genes that allow production of the bacteria in vitro under certain growth conditions but do not allow extended survival in vivo. Other attenuated organisms were generated by serial passage in broth or cultured cells and selection for reduction of virulence and maintenance of immunogenicity in animal models.

The manufacturing of live, attenuated organisms can be straightforward, as they often accumulate to high concentrations in bacterial

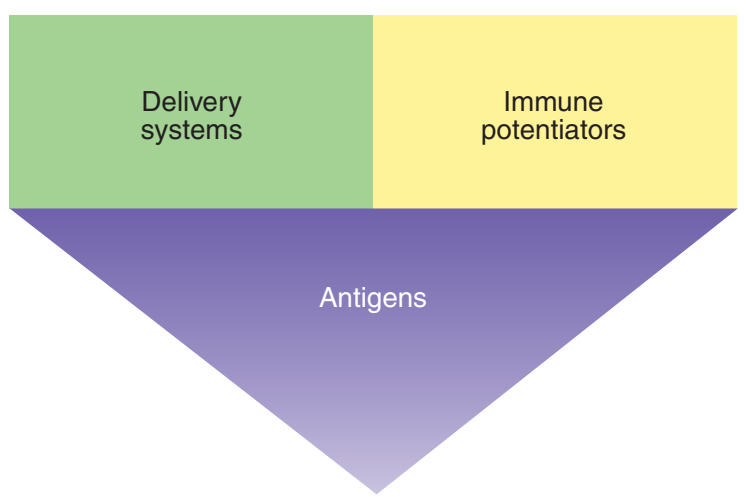

Figure 1 Key components of effective vaccines. Effective vaccines have three key components: (i) an antigen against which adaptive immune responses are generated, (ii) an immune stimulus or adjuvant to signal the innate immune system to potentiate the antigen-specific response, and (iii) a delivery system to ensure that the antigen and adjuvant are delivered together at the right time and location. Live, attenuated vaccines contain all of these elements, including most or all of the antigens of the pathogen, pathogen-associated molecular patterns that trigger innate immunity, and efficient delivery resulting from the natural invasiveness of the organism. culture media or supernatants of cells in which viruses are produced. As a consequence, purification yields and the number of vaccine doses produced can be high, thereby lowering the cost of the product. However, an important limitation of live organism-based vaccines is safety; it is not always possible to identify sufficiently attenuated strains, and there is a finite, underlying risk of reversion to virulence. For these reasons, progress toward live vaccines against certain chronic infections, such as with M. tuberculosis or HIV, has been slow.

Inactivated or killed organisms. A second general method of vaccine preparation is to inactivate or kill an otherwise pathogenic organism. In this way, all of the antigens of the organism are available to the immune system, but the organism itself is rendered harmless. This approach is most appropriate in cases where the organism does not possess overtly toxic components and can be inactivated without detriment to the fidelity of the antigens. Several viral and bacterial vaccines are produced in this manner, such as those against influenza virus, hepatitis A virus, rabies virus, and whole-cell Bordetella pertussis.

Because of their inability to replicate in vivo, killed vaccines are generally less immunogenic than live vaccines. To compensate, killed vaccines are often administered together with an adjuvant (such as aluminum salts) to increase potency. In addition, killed vaccines do not effectively elicit cell-mediated immunity (particularly cytotoxic $\mathrm{T}$ lymphocytes, or CTLs) because of a general lack of access of exogenous vaccine-administered antigens to the endogenous major histocompatibility complex class I antigen presentation pathway. However, killed vaccines are particularly useful for presenting conformational antibody epitopes found on the surface of the organism.

Manufacturing processes for killed organism-based vaccines are similar to those for live, attenuated vaccines. However, once purified, the live organisms are inactivated by treatment with chemical agents such as formaldehyde and $\beta$-propiolactone. Killed bacteria, such as 
Table 2 Changes in vaccine manufacturing

\begin{tabular}{|c|c|c|}
\hline Nature of change & Year & Reason \\
\hline Monovalent to combination vaccines (diphtheria, tetanus and pertussis) & 1940s & Reduce number of childhood immunizations \\
\hline Plasma-derived to recombinant hepatitis B & 1986 & Increase safety \\
\hline Free polysaccharides to conjugated $H$. influenzae $B$ & 1989 & Extend effectiveness to infants \\
\hline Whole-cell to acellular Pertussis & 1991 & Increase purity to reduce side effects \\
\hline Live, attenuated to inactivated polio & 1990 & Increase safety \\
\hline Inactivated to live, attenuated influenza & 2003 & Mucosal delivery, avoid use of needles \\
\hline Live, attenuated to reassorted rotavirus & 2006 & Increase safety \\
\hline Egg-derived to cell-cultured influenza & In development & Increase manufacturing speed and capacity \\
\hline Cell-filtrate to recombinant anthrax & In development & Increase potency, reduce side effects \\
\hline Outer membrane vesicle to recombinant meningococcus $B$ & In development & Broaden strain coverage \\
\hline
\end{tabular}

B. pertussis, are relatively uncharacterized biochemically and can be reactogenic, as they contain all the components of the bacteria. Killed viruses, in contrast, are usually shed into the cell culture supernatant and are thus relatively free of cellular components. Furthermore, the large size of virus particles compared to the other components of the culture medium enables a high degree of purification. These characteristics of killed organism-based vaccines have positioned this approach as the one of choice for induction of antibody-mediated protection against viral diseases.

Purified or recombinant subunit vaccines. Using entire live or killed organisms in vaccines eliminates the need to identify the protective antigens but has the potential disadvantages of safety and dilution or deviation of the protective immune responses. Thus, in cases where a protective antigen is known, it is often safer and more efficient to focus the immune response on this particular target.

Many bacteria, such as Clostridium tetani and Corynebacterium diphtheriae, produce toxins that lead to pathogenesis in the infected host. It has long been known that antibody-mediated neutralization of such toxins can prevent disease, and effective vaccines for this purpose are based on detoxified versions of these toxins (called toxoids).

Similarly, antibodies directed against the polysaccharides of certain capsular bacteria (for example, Neisseria meningitidis and Streptococcus pneumoniae) are known to exert antibacterial immunity. Vaccines against these organisms are therefore based on polysaccharides extracted and enriched from bacterial cultures. These polysaccharide vaccines are typically effective in adults but only poorly immunogenic in children less than 2 years old, a result of the immature immune systems in this age group and the $\mathrm{T}$ cell-independent nature of immune responses induced by polysaccharides. This deficiency has been overcome by the use of carrier proteins that provide helper T-cell responses and confer bystander $\mathrm{T}$ cell-dependent immunity against the polysaccharides. Vaccines against Haemophilus influenzae B, for example, are produced in this way by chemical conjugation of the purified polysaccharides to one of several different protein carriers.

Finally, recombinant protein-based vaccines produced in cell culture systems have been developed against hepatitis B virus and the causative agent of Lyme disease, Borrelia burgdorferi, and several others are in development (discussed below).

\section{Changes in vaccine manufacturing}

The impetus for changes in the way certain vaccines are designed or manufactured has been driven by several factors, including the need to improve the potency, quality or safety of a vaccine; or practical reasons such as ease or cost of manufacture. Many vaccines have undergone changes in the way they are manufactured (Table 2). Following are selected examples of why changes have been or are in the process of being made.

Recombinant protein vaccines. The main drivers for developing recombinant subunit proteins to replace existing vaccines center on developing less reactogenic, more potent vaccines (for example, anthrax vaccine); developing safer, better characterized vaccines (for example, hepatitis $B$ virus vaccine); and developing vaccines that provide broader protection against multiple serotypes of a bacterium (for example, N. meningitidis $B$ ).

The current licensed anthrax vaccine is prepared as a cell-free filtrate from cultures of an attenuated strain of Bacillus anthracis, without further purification. The process is straightforward, but the vaccine is relatively uncharacterized and contains many constituents shed from the bacteria that are likely to contribute to the morbidity sometimes associated with anthrax vaccination. In contrast, a next-generation anthrax vaccine currently being tested in human clinical trials is a highly purified protective antigen produced in bacterial cell culture

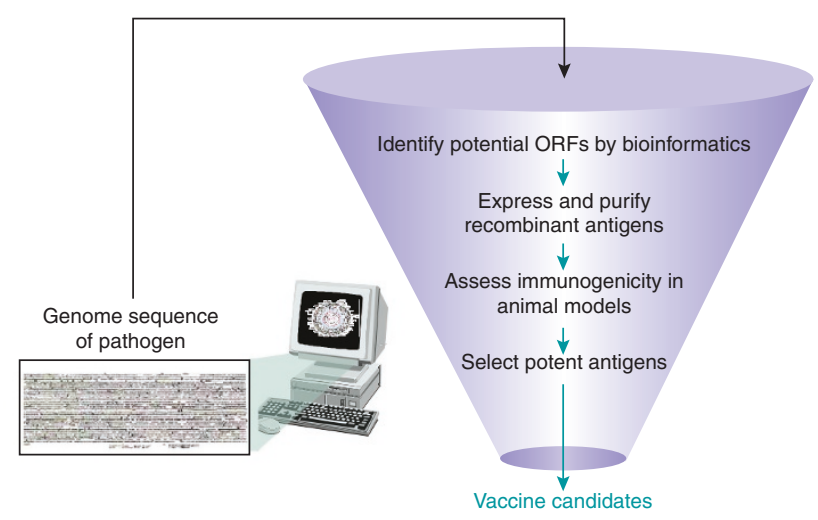

Figure 2 Reverse vaccinology for identification of novel vaccine antigens. Reverse vaccinology is a new technology borne from a need to identify novel vaccine antigens for meningococcus serogroup $B$. From genome sequence information, all potential open reading frames (ORFs) are identified using bioinformatics approaches. Recombinant DNA techniques are used to express these gene products in $E$. coli. The resulting proteins are tested in animal models; those proteins that are able to elicit functional antibody responses are potential vaccine candidates. This approach is a powerful means to rapidly identify novel protein antigens from an organism in an unbiased manner. 


\section{Box 1 Gap between supply and demand of pandemic influenza vaccines}

The seasonal trivalent influenza vaccines contain $15 \mu \mathrm{g}$ of hemagglutinin antigen for each of three virus strains ( $45 \mu \mathrm{g}$ of hemagglutinin total). The current worldwide annual capacity for production of seasonal egg-based vaccine is approximately 1 billion doses of monovalent vaccine (amounting to $>300$ million doses of seasonal trivalent vaccine), including a 3-month lead time for start of production. Assuming the optimistic scenario that a pandemic influenza vaccine will consist of a single dose of $15 \mu \mathrm{g}$ of hemagglutinin from a single virus strain, at this capacity only $\sim 40 \%$ of the world's population would be covered in 2 years, and it would take almost 5 years with $>2$ billion eggs to produce sufficient quantities of vaccine for everyone ( 1 egg yields $\sim 3$ monovalent doses; see Table 3). Modeling scenarios for a potential pandemic in the US have shown that vaccination would be the most effective
Table 3 Scale-up of influenza vaccine production

\begin{tabular}{ccc}
$\begin{array}{c}\text { Production time } \\
\text { (including lead time) }\end{array}$ & $\begin{array}{c}\text { Worldwide capacity } \\
\text { (monovalent doses of } \\
\mathbf{1 5} \boldsymbol{\mu g} \text { ) }\end{array}$ & $\begin{array}{c}\text { Worldwide coverage } \\
\text { (\%) }\end{array}$ \\
\hline 1 year & $\sim 1,000,000,000$ & $\sim 17$ \\
2 years & $\sim 2,500,000,000$ & $\sim 40$ \\
4 years and 9 months & $\sim 6,500,000,000$ & 100 \\
\hline
\end{tabular}

means of reducing the number of casualties, provided a vaccine could be produced and distributed quickly ${ }^{36}$. Thus, a rapid scaleup of capacity by a factor of 6 would be needed to ensure vaccine supply for the world's population within 1 year. This is currently not possible using egg-based production because of lack of manufacturing infrastructure and egg availability. using recombinant DNA technology ${ }^{6,7}$. The safety and efficacy of this vaccine in humans remains to be fully evaluated, but has the potential to offer superiority over the current anthrax vaccine, which requires a series of six immunizations followed by annual boosters.

Current vaccines for meningitis B are based on crude membrane preparations, termed outer membrane vesicles, which contain several 을 bacterial surface antigens that induce bactericidal antibodies. These vaccines are potent but do not provide broad coverage across the many serogroups of $N$. meningitidis. In some cases where the local disease is caused by a small number of circulating strains, such as in Norway, outer membrane vesicle vaccines have been effective in controlling disease $^{8}$. However, in most parts of the world, a better vaccine providing broader coverage is needed. Recently, a comprehensive screening of the genome of the microorganism (an approach termed 'reverse vaccinology') was undertaken to identify protective protein antigens, resulting in a candidate vaccine consisting of several N. meningitidis proteins ${ }^{9}$ (Fig. 2). This vaccine has been effective in animal models at inducing bactericidal antibodies capable of recognizing a broad spectrum of $N$. meningitidis serogroups ${ }^{10}$ and is currently being evaluated in human clinical trials. However, the vaccine is administered as a combination of recombinant proteins that require separate manufacture in Escherichia coli. One potential strategy to simplify the manufacturing process is to express some of the proteins as polyprotein fusion constructs, thereby reducing the number of required products while retaining immunogenicity against all of the components. This approach is currently being evaluated for other vaccines ${ }^{11}$.

The move toward fully recombinant vaccines produced in cell culture has resulted in products with more demanding manufacturing and downstream processing. These challenges may be contributing to the lack of introduction of many new vaccines based on recombinant proteins. However, the extra manufacturing and processing steps will be justified if the resulting vaccines provide the safety and efficacy benefits described above.

Improved influenza vaccines. Influenza vaccines based on virus grown in and isolated from embryonated chicken eggs and then inactivated have been widely used for more than 60 years. Enhancements in manufacturing have been made by improving the purification process to remove reactogenic components. In addition, high-yield reassortant strains have been developed, consisting of the hemagglutinin and neuraminidase genes from the circulating influenza strain and the remaining genes derived from a standard influenza virus strain optimized for production in eggs. Despite these improvements, the basic vaccine strategy has not changed over this time, primarily because the low profit margins provided by the influenza vaccine product did not support investment in new production technologies. However, the recent increase in demand for the vaccine and the threat of a pandemic have accelerated the introduction of new manufacturing strategies.

Limitations of the current influenza vaccine include issues of effectiveness, tolerability and manufacturing. For example, the level and duration of immune responses induced by the inactivated-virus vaccine, while usually adequate for healthy adults during a single influenza season, could be improved to provide prolonged protective immunity from one season to the next and in children and the elderly. In addition, inactivated vaccines administered parenterally are not effective at inducing mucosal or cellular immune responses, particularly CTLs, which may be necessary for optimum protection against this respiratory pathogen. In contrast, immunity against influenza acquired by natural infection is effective and thought to be mediated by multiple effector mechanisms, including serum antibodies, mucosal immune responses and CTLs. Immunity generated this way is long-lived against exposure to homologous viruses and provides some measure of protection against antigenically drifted virus strains.

The perception of side effects and fear of needle injections are reasons for low immunization rates with the influenza vaccine. A better-tolerated vaccine administered by a less invasive route could persuade more people to be vaccinated. A live, attenuated influenza virus vaccine delivered to mucosal surfaces may, in principle, be able to achieve similar or better protection compared to inactivated vaccine without the use of needles. In 2003, a cold-adapted influenza vaccine (CAIV; FluMist, MedImmune Vaccines) was licensed in the United States for intranasal administration to healthy individuals 5-49 years of age. The original cold-adapted virus strain was generated by serial passage at $25^{\circ} \mathrm{C}$ in tissue culture cells, resulting in attenuation. The temperature-sensitive nature of CAIV restricts virus replication to the mucosa of the upper respiratory tract after intranasal inoculation, thereby largely eliminating reactogenicity. CAIV induces serum IgG and nasal IgA antibodies as well as T-cell responses and can provide protection against antigenically drifted influenza virus strains in humans ${ }^{12}$. The segmented genome of influenza viruses facilitates production of the vaccine strains for both conventional vaccine and CAIV by allowing reassortment of the hemagglutinin and neuraminidase genes derived from the circulating viruses, recommended for inclusion in the vaccine, together with the remaining six genes from the attenuated master donor virus. A potential concern for manufacturing of any live vaccine is genetic 


\section{Box 2 Existing and targeted supply of pandemic influenza vaccine for the world}

Reliable numbers on worldwide planned cell culture-based influenza production are not yet available. However, the United States Department of Health and Human Services recently granted contracts to six vaccine manufacturers with the goal of creating capacity for production of 600 million doses of pandemic vaccine in a 6-month period (including lead time). Subsequent production for an additional 6 months would result in $\sim 1.45$ billion doses of cell culture-based vaccine in a 1-year period (Table 4). This resulting yearly cell culture capacity for the United States alone would be higher than the existing worldwide egg-based production capacity. Together with current worldwide egg-based capacity, it

\begin{tabular}{|c|c|c|c|}
\hline & $\begin{array}{c}\text { Existing } \\
\text { worldwide egg-based } \\
\text { vaccine capacity }\end{array}$ & $\begin{array}{l}\text { Targeted US } \\
\text { cell-culture capacity }\end{array}$ & $\begin{array}{c}\text { Total } \\
\text { capacity }\end{array}$ \\
\hline $\begin{array}{l}\text { 12-month capacity } \\
\text { (including lead time) }\end{array}$ & 1,000,000,000 & $\sim 1,450,000,000$ & $\sim 2,450,000,000$ \\
\hline
\end{tabular}

would take only a 2.5 -fold increase in this cell culture-based influenza production capacity outside of the United States to deliver sufficient pandemic vaccine to cover worldwide demand within a 1year period. Enhancements to potency to reduce the dose of vaccine required for protective immunity, such as the use of adjuvants, will decrease the time required for meeting worldwide demand. instability during production. A recent study, however, has shown that no mutations occurred in any of nine CAIVs evaluated during the manufacturing process ${ }^{13}$. Like the inactivated virus vaccine, though, production of CAIV currently takes place in eggs.

The manufacturing processes to produce current influenza vaccines are time consuming and labor intensive and involve millions of eggs, thus requiring long lead times for vaccine production (Boxes 1 and 2 and Tables 3 and 4). As a consequence, the choice of influenza virus strain to be used in the vaccine must be made many months in advance of the influenza season, which increases the risk of having a mismatch between the vaccine and the circulating virus strains. Furthermore, growth of influenza virus in eggs has the potential to select for variants that grow well in those conditions ${ }^{14}$, leading to further mismatch with circulating viruses. Clearly, a more rapid and technologically advanced manufacturing process is needed.

One solution to this problem is to avoid eggs altogether by growing the vaccine virus in tissue culture cells. In this way, the manufacturing process will be more rapid, better controlled and more easily increased for large-scale vaccine production (as would be needed to supply vaccine worldwide in the case of a pandemic; Box 2 ). In addition, the issue of potential allergic reactions to egg components would be eliminated by the use of tissue-culture production systems. Approaches to producing influenza vaccine virus in Vero cells, Madin-Darby kidney cells and PER.C6 cells are showing promise. For example, virus produced in Madin-Darby kidney cells does not seem to select for variants distinct from the virus circulating in humans ${ }^{15}$ and is at least as potent as eggderived vaccine in animal models and humans ${ }^{16}$. Influenza vaccines produced in cell culture are in late-stage development and should eventually replace egg-grown vaccines in the marketplace.

Further advancements in the use of cell lines will include reverse genetics techniques, where RNA genes from circulating viruses are reverse-transcribed into DNA. These plasmid DNAs encoding the influenza antigens are then used to transfect cells as a means to generate vaccine viruses ${ }^{17,18}$, rather than using live virus to produce the vaccine. This approach has the potential to streamline the cumbersome and inefficient process of generating reassortant viruses by coinfection of eggs or cells. The use of cell lines and reverse genetics to more quickly manufacture many doses of vaccine will be particularly important in response to newly emerging influenza viruses, such as the avian $\mathrm{H} 5 \mathrm{~N} 1$ strains currently circulating, that have the potential to cause widespread disease in humans. In addition, a reduction in the lag between identification of circulating virus strains and availability of vaccine will decrease the chances of a mismatch.

\section{New vaccine technologies}

As described above, there have been many successes with vaccines based on live or killed organisms and components thereof. However, these traditional approaches have not been effective for the many vaccine challenges that remain, such as preventing or treating chronic infections and cancer. These situations may require induction of potent cellular immune responses, such as CTLs, to facilitate clearance of infected or neoplastic cells. In addition, a more specific focus on key antigens may be needed, particularly for targeting neoantigens on tumors. New approaches to the way vaccine antigens are presented and delivered to the immune system will be required to achieve these types of responses and to break immunologic tolerance against self antigens (important in cancer). Several potentially enabling technologies are under development and may have broader implications for the manufacture of existing vaccines.

Gene-based vaccines. Cellular immunity, particularly the CTL response, is usually efficiently induced when the antigen is expressed by host cells, such as during a viral infection, to allow appropriate antigen presentation by the immune system. Vaccines based on live organisms have the ability to elicit such responses, as do gene-based vaccines consisting of plasmid DNA or recombinant viral vectors. Certain particle-based vaccine formulations, such as VLPs, also have the potential to induce CTLs (see below) through internalization and processing by antigenpresenting cells, in a manner distinct from the endogenous pathway described earlier for live-virus vaccines. One attractive feature of genebased vaccines is the potential to combine the simplicity and focused immune responses provided by subunit vaccines with the CTL induction provided by live organisms ${ }^{19}$. Because of these attributes, genebased vaccines have shown promise in preclinical animal models and human clinical trials of infectious disease and cancer vaccines ${ }^{20-23}$.

From a practical perspective, these vaccines may facilitate manufacturing as well. First, plasmid DNA vaccines are produced in E. coli using standard fermentation and downstream processing techniques that are amenable to large-scale production and yield a highly pure and stable vaccine. A recent analysis has suggested that existing manufacturing technologies and infrastructure have the potential to provide DNA vaccines on a global scale ${ }^{24}$. Second, recombinant DNA technologies may facilitate the speed of manufacturing new vaccines, which may be particularly important for newly emerging diseases such as pandemic influenza. The only distinction between DNA vaccines, regardless of disease target, is the sequence of the gene insert. Thus, the processes to manufacture these vaccines are generic and likely to be independent 
of the nature of the DNA vaccine. The prospects for this technology are promising, as two DNA vaccine products were licensed in 2005 for animal health applications ${ }^{23}$.

However, DNA vaccines administered in buffer by needle injection (termed "naked DNA") have so far shown only modest immunogenicity in humans. Hence, facilitated delivery technologies may be required for increased DNA vaccine potency. As an alternative to plasmid DNA, gene-based vaccines consisting of recombinant viral vectors derived from adenovirus, poxviruses and alphaviruses are being investigated for various vaccine applications. Like DNA vaccines, recombinant viral vectors deliver genes encoding vaccine antigens, but with much more efficiency. Where comparisons have been made, immunogenicity studies have indicated that viral vector vaccines induce stronger immune responses in humans than do DNA vaccines ${ }^{25}$. However, from a manufacturing perspective, viral vector-based vaccines are likely to be more complicated than DNA vaccines. Vector design, genetic stability, producer cell lines, conditions of virus propagation and purification and product characterization all need to be established to ensure highquality, safe products. Several such vectors are currently being evaluated in human clinical trials, so these issues are being addressed.

Aside from these technical challenges of manufacturing, it has been well established that preexisting or vector-induced immunity can limit the effectiveness of certain viral vector vaccines (such as those using adenovirus and poxvirus vectors). This major drawback must 을 be overcome to realize the potential for broad use of this type of vaccine. Strategies to circumvent this potential problem include the use of higher doses, rare serotypes to which most people have not been exposed, heterologous prime-boost regimens involving different vaccine modalities, and vector engineering to remove immunodominant epitopes.

VLPs. VLPs are formed by self-assembly of viral capsid proteins upon expression in cell culture systems. VLP-based vaccines possess several potential advantages over other types of vaccines. First, they are particulate, contain many copies of antigen in a repeating array and present conformational antibody epitopes, thereby mimicking viral surface structure. These properties render VLPs very efficient for induction of both humoral and cell-mediated immunity, includ-

ing CTLs. Second, they do not package viral nucleic acids and are thus nonreplicating and very safe. Third, they have been shown to be effective when administered mucosally, including orally. Finally, VLPs can be produced in a variety of expression systems, such as mammalian, insect, yeast, bacteria and plant cells. This provides flexibility in tailoring manufacturing conditions to the specific needs of the product, such as the requirement for mammalian posttranslational processing.

The efficient self-assembly of certain viral capsids and the particulate nature of VLPs greatly facilitates production and purification of this type of vaccine compared to soluble recombinant protein vaccines. Several viral antigens have been expressed as VLPs, including those from HIV, Norwalk virus and HPV, all of which have been tested in human clinical trials. The HPV VLP vaccine, based on the L1 antigen from HPV16 and HPV18, has been shown to be immunogenic and highly protective against infection and associated cervical lesions in human clinical trials ${ }^{26}$ and was recently licensed for human use. The effectiveness of this vaccine is likely to pave the way for other VLPbased vaccines, as well as the use of VLPs as carrier systems for delivery of other antigens. For example, a malaria vaccine candidate (termed repeat T epitope, S antigen, or RTS,S) consisting of the circumsporozoite protein antigen grafted onto the hepatitis B core capsid has shown immunogenicity and efficacy in clinical trials ${ }^{27}$.
Plant-derived vaccines. The rationale for an oral vaccine derived from plants is to improve delivery and production. Given that most pathogens gain entry into their hosts via mucosal surfaces, the induction of a robust mucosal immune response by vaccination may be an effective means of preventing infectious diseases. Oral delivery of vaccines is particularly appealing, as it would avoid needles and allow self-administration. Vaccine production in plants is attractive in terms of safety and cost effectiveness. From a safety perspective, the plant expression system would produce a vaccine free of animal pathogens and animal proteins. This would eliminate the risk of contamination by animal materials containing mutant prions and other transmissible agents, thereby providing a significant advantage over other recombinant expression systems that rely on the use of animal-derived materials for production. The production of plant-derived vaccines is, in principle, almost limitless and may require little or no downstream processing. Transgenic plants such as potato, tomato and banana expressing vaccine antigens could be grown at agricultural scale to yield ready-to-eat vaccines without the need for complex and costly manufacturing procedures and facilities.

So far, transgenic potatoes expressing a bacterial toxin and antigens from the hepatitis B and Norwalk viruses have been shown to be immunogenic as raw, edible vaccines in separate phase 1 clinical trials ${ }^{28,29}$. Challenges that remain include understanding the potential for inducing oral immunologic tolerance, developing oral adjuvants that may be necessary to increase vaccine potency, controlling the dose for self-administered oral vaccines, determining the impact of plant glycosylation patterns on immunogenicity and identifying the optimal plant expression system. With regard to the latter, achieving high levels of antigen expression in a plant that can be eaten raw may be important to preserve antigen structure.

Novel adjuvants and delivery systems. Vaccines consisting of recombinant or purified subunits typically lack an immune stimulus and means of delivery, in contrast to whole-organism vaccines, which contain many immunostimulatory components and are naturally invasive. Thus, subunit vaccines usually require added adjuvants and delivery systems for potency. So far, only a few such additives are licensed for use with human vaccines, including aluminum salts and the oil-in-water emulsion MF59. However, recent insight into the workings of the innate immune system has highlighted the crucial role of innate immune stimulation in the maturation of an adaptive immune response. It is now known that signaling of one or more receptors on immune cells (for example, Toll-like receptors) results in a rapid inflammatory response, leading to enhanced presentation of antigens to the immune system. Much effort is therefore under way to harness the innate immune system for the purposes of increasing or modulating immune responses to vaccines ${ }^{30}$.

To this end, several experimental immune potentiators are in development and have been shown to have profound effects on vaccine potency. These include immunostimulatory CpG oligonucleotides ${ }^{31}$, synthetic analogs of monophosphoryl lipid $\mathrm{A}^{32}$, and the small molecule imidazoquinoline compounds $s^{33}$, all of which signal the innate immune system. Simple mixtures of these immune potentiators with vaccines can be effective, although in most cases the adjuvant effect can be substantially enhanced through the use of a delivery system. For example, microparticles and emulsions have been used to codeliver vaccine antigens and immune potentiators, resulting in markedly enhanced vaccine potency in animal models ${ }^{34}$.

Such vaccines, though, may pose manufacturing complications, in that as many as three distinct components must be produced separately and then formulated into the final product. The antigens would be 
produced in the same manner as other subunit vaccines, but additional processes for the immune potentiator and delivery system will be required. For small-molecule immune potentiators, large-scale synthesis should not be a limitation, as this type of manufacturing is routine for small-molecule pharmaceutical drugs. In the case of the imidazoquinolines, a product is already on the market for the topical treatment of external genital warts, superficial basal-cell carcinoma and actinic keratosis ${ }^{35}$. Despite the complication of multiple components, such vaccines are therefore feasible from a manufacturing standpoint and would be warranted in instances where conventional vaccine technologies are not effective.

\section{Conclusions}

Several vaccines in use today were originally introduced many years ago but have undergone refinement over time (Table 2). Factors that have driven modification of these vaccines include the need for improved vaccine safety, tolerability and potency. The result has been better products but also more demanding manufacturing processes, which are justified by the benefits provided by the improved vaccines. Other vaccines that have been available for many decades, such as influenza vaccine, are still being produced by rather antiquated technologies. Changes in the way these vaccines are being made are driven by the desire to use more efficient and technologically advanced manufacturing processes. The result will be vaccines equivalent to or better than 음 their predecessors but produced with state-of-the-art methods and at a scale and speed to meet demands. Finally, there are vaccines yet to be developed against diseases where traditional approaches have not been successful. In these cases, new enabling technologies will be needed, some of which may also have an impact on the way existing vaccines are manufactured. Despite these advances, long-standing challenges will remain or intensify. These include overcoming the inertia to change well-established manufacturing procedures, addressing complex regulatory issues associated with introducing new types of vaccine products for healthy people, and managing the increased costs to produce complicated new vaccines.

\section{COMPETING INTERESTS STATEMENT}

The authors declare competing financial interests (see the Nature Biotechnology website for details).

Published online at http://www.nature.com/naturebiotechnology/

Reprints and permissions information is available online at

http://npg.nature.com/reprintsandpermissions/

1. Cooper, M.R., Stewart, D.C., Kahl, F.R., Brown, W.M. \& Cordell, A.R. Medicine at the medical center then and now: one hundred years of progress. South. Med. J. 95, 1113-1121 (2002).

2. Sheridan, C. The business of making vaccines. Nat. Biotechnol. 23, 1359-1366 (2005).

3. Greco, M. Key drivers behind the development of global vaccine market. Vaccine 19, 1606-1610 (2001).

4. Martineau, B. Stats. Sticking with growth. Vaccine market continues to rise. Mater. Manag. Health Care 13, 42 (2004).

5. Rappuoli, R., Miller, H.I. \& Falkow, S. Medicine. The intangible value of vaccination. Science 297, 937-939 (2002).

6. Farchaus, J.W., Ribot, W.J., Jendrek, S. \& Little, S.F. Fermentation, purification, and characterization of protective antigen from a recombinant, a virulent strain of Bacillus anthracis. Appl. Environ. Microbiol. 64, 982-991 (1998).

7. Ribot, W.J. et al. Comparative vaccine efficacy of different isoforms of recombinant protective antigen against Bacillus anthracis spore challenge in rabbits. Vaccine 24, 3469-3476 (2006)

8. Holst, J. et al. Serum bactericidal activity correlates with the vaccine efficacy of outer membrane vesicle vaccines against Neisseria meningitidis serogroup B disease. Vaccine 21, 734-737 (2003)

9. Tettelin, $\mathrm{H}$. et al. Complete genome sequence of Neisseria meningitidis serogroup $\mathrm{B}$ strain MC58. Science 287, 1809-1815 (2000).

10. Pizza, M. et al. Identification of vaccine candidates against serogroup $B$ meningococcus by whole-genome sequencing. Science 287, 1816-1820 (2000).

11. Langermans, J.A. et al. Protection of macaques against Mycobacterium tuberculosis infection by a subunit vaccine based on a fusion protein of antigen $85 \mathrm{~B}$ and ESAT-6. Vaccine 23, 2740-2750 (2005).

12. Belshe, R., Lee, M.S., Walker, R.E., Stoddard, J. \& Mendelman, P.M. Safety, immunogenicity and efficacy of intranasal, live attenuated influenza vaccine. Expert Rev. Vaccines 3, 643-654 (2004).

13. Buonagurio, D.A. et al. Genetic stability of live, cold-adapted influenza virus components of the FluMist/CAIV-T vaccine throughout the manufacturing process. Vaccine 24, 2151-2160 (2006).

14. Hardy, C.T., Young, S.A., Webster, R.G., Naeve, C.W. \& Owens, R.J. Egg fluids and cells of the chorioallantoic membrane of embryonated chicken eggs can select different variants of influenza A (H3N2) viruses. Virology 211, 302-306 (1995).

15. Katz, J.M. \& Webster, R.G. Amino acid sequence identity between the HA1 of influenza A (H3N2) viruses grown in mammalian and primary chick kidney cells. J. Gen. Virol. 73, 1159-1165 (1992).

16. Halperin, S.A. et al. Safety and immunogenicity of a trivalent, inactivated, mammalian cell culture-derived influenza vaccine in healthy adults, seniors, and children. Vaccine 20, 1240-1247 (2002).

17. Nicolson, C., Major, D., Wood, J.M. \& Robertson, J.S. Generation of influenza vaccine viruses on Vero cells by reverse genetics: an $\mathrm{H} 5 \mathrm{~N} 1$ candidate vaccine strain produced under a quality system. Vaccine 23, 2943-2952 (2005).

18. Neumann, G., Fujii, K., Kino, Y. \& Kawaoka, Y. An improved reverse genetics system for influenza $A$ virus generation and its implications for vaccine production. Proc. Natl. Acad. Sci. USA 102, 16825-16829 (2005).

19. Donnelly, J.J., Ulmer, J.B., Shiver, J.W. \& Liu, M.A. DNA vaccines. Annu. Rev. Immunol. 15, 617-648 (1997)

20. Wang, R. et al. Induction of antigen-specific cytotoxic $T$ lymphocytes in humans by a malaria DNA vaccine. Science 282, 476-480 (1998).

21. Rottinghaus, S.T., Poland, G.A., Jacobson, R.M., Barr, L.J. \& Roy, M.J. Hepatitis B DNA vaccine induces protective antibody responses in human non-responders to conventional vaccination. Vaccine $21,4604-4608$ (2003).

22. McShane, H. et al. Recombinant modified vaccinia virus Ankara expressing antigen $85 \mathrm{~A}$ boosts BCG-primed and naturally acquired antimycobacterial immunity in humans. Nat. Med. 10, 1240-1244 (2004).

23. Ulmer, J.B., Wahren, B. \& Liu, M.A. Gene-based vaccines: recent technical and clinical advances. Trends Mol. Med. 12, 216-222 (2006).

24. Hoare, M. et al. Bioprocess engineering issues that would be faced in producing a DNA vaccine at up to $100 \mathrm{~m} 3$ fermentation scale for an influenza pandemic. Biotechnol. Prog. 21, 1577-1592 (2005).

25. McConkey, S.J. et al. Enhanced T-cell immunogenicity of plasmid DNA vaccines boosted by recombinant modified vaccinia virus Ankara in humans. Nat. Med. 9, 729-735 (2003).

26. Harper, D.M. et al. Sustained efficacy up to 4.5 years of a bivalent L1 virus-like particle vaccine against human papillomavirus types 16 and 18: follow-up from a randomised control trial. Lancet 367, 1247-1255 (2006).

27. Alonso, P.L. et al. Duration of protection with RTS,S/ASO2A malaria vaccine in prevention of Plasmodium falciparum disease in Mozambican children: single-blind extended follow-up of a randomised controlled trial. Lancet 366, 2012-2018 (2005).

28. Tacket, C.O. Plant-derived vaccines against diarrheal diseases. Vaccine 23, 1866-1869 (2005).

29. Thanavala, Y. et al. Immunogenicity in humans of an edible vaccine for hepatitis $B$. Proc. Natl. Acad. Sci. USA 102, 3378-3382 (2005).

30. Pashine, A., Valiante, N.M. \& Ulmer, J.B. Targeting the innate immune response with improved vaccine adjuvants. Nat. Med. 11, S63-S68 (2005).

31. Cooper, C.L. et al. CPG 7909, an immunostimulatory TLR9 agonist oligodeoxynucleotide, as adjuvant to Engerix-B HBV vaccine in healthy adults: a double-blind phase $1 / 1$ study. J. Clin. Immunol. 24, 693-701 (2004).

32. Vandepapeliere, P. et al. Potent enhancement of cellular and humoral immune responses against recombinant hepatitis B antigens using ASO2A adjuvant in healthy adults. Vaccine 23, 2591-2601 (2005).

33. Wille-Reece, U., Wu, C.Y., Flynn, B.J., Kedl, R.M. \& Seder, R.A. Immunization with HIV-1 Gag protein conjugated to a TLR7/8 agonist results in the generation of HIV-1 Gag-specific Th1 and CD8+ T cell responses. J. Immunol. 174, 7676-7683 (2005).

34. Kazzaz, J. et al. Encapsulation of the immune potentiators MPL and RC529 in PLG microparticles enhances their potency. J. Control. Release 110, 566-573 (2006).

35. Vidal, D. Topical imiquimod: mechanism of action and clinical applications. Mini Rev. Med. Chem. 6, 499-503 (2006).

36. Germann, T.C., Kadau, K., Longini, I.M., Jr \& Macken, C.A. Mitigation strategies for pandemic influenza in the United States. Proc. Natl. Acad. Sci. USA 103, 5935-5940 (2006). 